\title{
Some Comments on the Real Meaning of Schrödinger's Equation Revealed by the Fact that Electron Is Always a Particle
}

\author{
Hironari Yamada \\ Faculty of Science and Engineering, Ritsumeikan University, Kusatsu, Japan \\ Email: hironari@se.ritsumei.ac.jp
}

Received October 7, 2012; revised November 8, 2012; accepted November 16, 2012

\begin{abstract}
We all physicist have long been believed that an elementary particle is a wave as well as a particle, but we discuss in this paper that an electron (probably all fermions) is always a particle. Author claim that quantum mechanics (QM) is not such mysterious as Bohr stated that the wave turn to the particle by observation. We can understand QM by natural human sense. The wave nature of electrons is only an appearance or a phenomena but not intrinsic or substantial. An electron is an individual body, which interferes with other individual electrons. Interference is the key word instead of the wave to understand the quantum mechanics. Interference produces the wave nature and the uncertainty. When we determine that an electron is nothing but a particle, we will see the true meaning of wave function and the Schrödinger's equation.
\end{abstract}

Keywords: Quantum Mechanics; Interference; Uncertainty Principle; Pauli Exclusive Principle; Schrödinger’s Equation

\section{Introduction}

There is no doubt on the wave nature of elementary particles, since Schrödinger's equation was so powerful to reveal the nature in the microscopic world last 90 years. As Nils Bohr, however, stated that "an electron immediately shrinks to a particle from a wave by observing, it created great confusion to the meaning of existence. The observing is an action by human. If we read this statement correctly we human can change the nature of elementary particle. If the nature is changed by observing, we lose our foundation of physics. Einstein and Schrödinger wondered this Bohr's statement and offered another opportunity of thinking in EPR [1] and Schrödinger's cat [2]. If we follow the experimental results on EPR it turned out that Einstein is wrong [3], but this doesn't automatically mean that Bohr is correct.

Recent empirical result by Hasegawa [4] raised the question that the Heisenberg's uncertainty principle [5] is not applicable to neutron or electron propagating free space. The momentum and the position are measured precisely and simultaneously. We think that it is the time to think over the foundation of quantum mechanics. We discuss in this paper the meaning of uncertainty, the meaning of wave, the meaning of observation and so on, and conclude that electron has a kind of will as Conway proposed the word free will [6].
Author claims in this paper that Fermions can be always a particle and that the wave nature appears only when electron is captured in a potential. Wave nature appears by the interference between elementary particles.

\section{No Explicit Evidences Indicating the Electron Wave}

\subsection{Experiment by Don Eigler}

If we look at carefully the past experimental results we see there are no evidences of electron being a wave. You might think that there is a beautiful demonstration of electron wave as show in Figure 1. This picture is obtained by Don Eigler [7] which is measured by an atomic force microscope (AFM). The 48 As atoms are circularly deposited on a $\mathrm{Cu}$ coated wafer like a coral reef. The wave structure appears inside the coral reef which is similar to water's standing wave in a circular pan. Author, however, thinks that this is not the evidence of one electron wave. AFM measures the density distribution of electrons. This structure is not made of one electron, but is made of many electrons. Yes wave nature appears as a statistics. This wave nature is very similar to that of water pun. We know that the water's ripple is a nature of water surface, but nobody feels so that water or $\mathrm{H}_{2} \mathrm{O}$ molecule is a wave. 


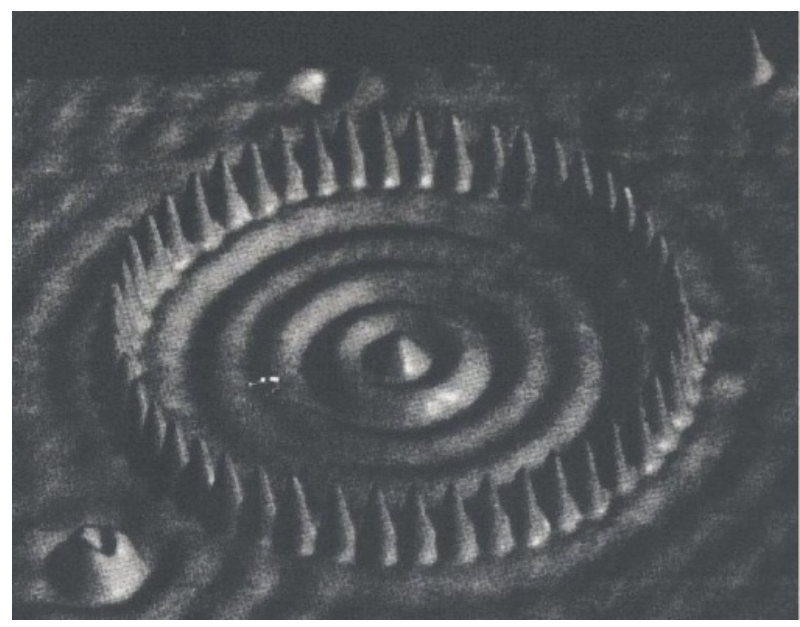

Figure 1. The 48 As atoms are circularly deposited on a $\mathrm{Cu}$ coated on a wafer like a coral reef, named quantum corral. The wave structure appears inside the coral reef which is similar to water's standing wave in a circular pan. But author thinks that this is not the evidence of one electron wave.

\subsection{Experiment by Tonomura}

Another example, Figure 2(a), is demonstrated by Tonomura [8]. The experimental setup of this measurement is illustrated in Figure 2(b). Electrons are emitted one by one from the top of thin needle shaped cathode. Electron propagates through two separated passes. Then finally each electron stains a spot on a two dimension digital camera. Here each electron appears as a spot (see in the right low) which indicates a particle. When the number of electrons is increased interference pattern appears as a whole nature. Here again, the interference pattern appears by the statistical nature. We don't see again the evidence of each electron being a wave. Tonomura states that each time only one electron is ejected, so this electron must pass both slits simultaneously. Thus electron is a wave, and turned to the particle when the observation is made as Bohr stated. People think that we can't understand the quantum mechanical phenomena by our normal human sense. Author will give a correct interpretation soon.

\section{Pauli Exclusion Principle Is an Evidence of Fermion Being an Individual Particle}

Pauli exclusion principle [9] is a strict evidence of Fermions' particle nature but not wave nature. All elementary particles are classified into either Fermion or Boson depending on the symmetry of the wave function and spin. Fermion has a half integer spin, thus the wave function is asymmetry. Boson has an integer spin, and the wave function is symmetry. It is well known that Fermion follows the Pauli exclusion principle but not for Boson. More than two Fermions can't stay in the exact
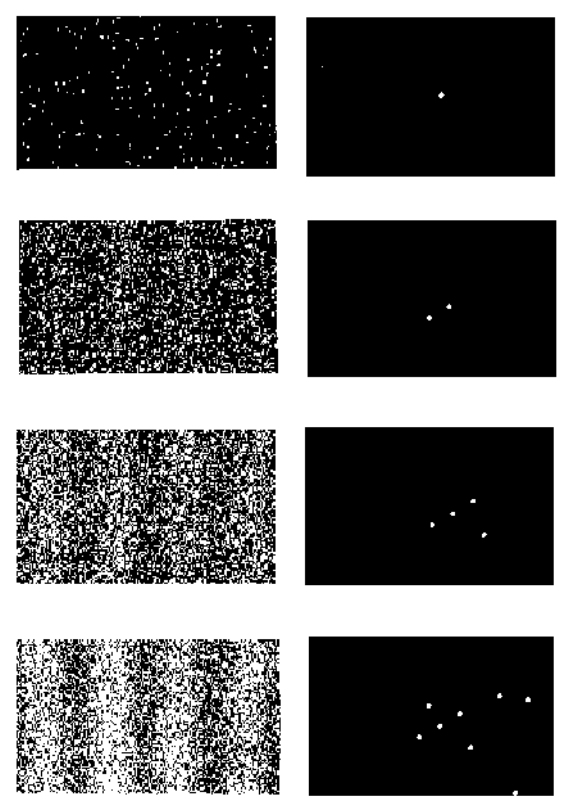

(a)

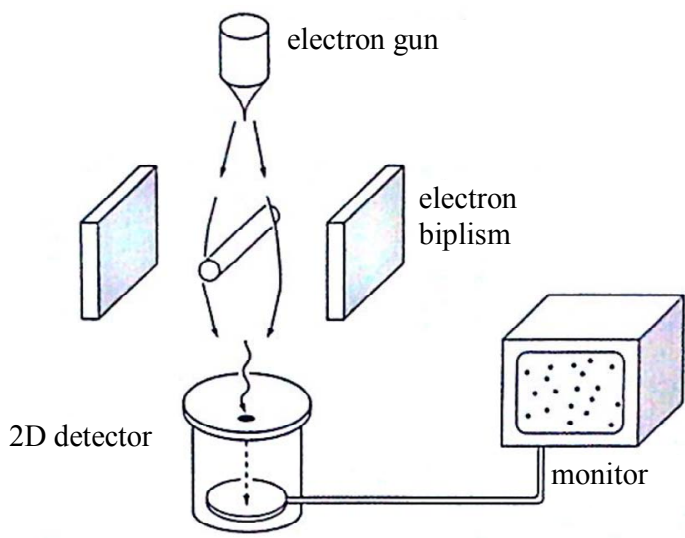

(b)

Figure 2. Tonomura's experiment. (a) Each electron makes a stain one by one on a $2 \mathrm{D}$ detector. When the number is increased interference pattern appears; (b) These stain is observed when electron pass through the double slit or byplism.

same phase space and level, but Boson can. Pauli princeple is here interpreted in the framework of wave function, but other interpretation is possible.

Fermion can be a particle in the sense of an individual $[10,11]$. Since it is an individual thus is exclusive. Every individual's existence is exclusive. We understand naturally that more than two particles can't stay in the exact same phase space, because they are individuals. We can say that every individual for instance human, cell, DNA, molecule, atom etc. follows the Pauli exclusion principle.

Let's consider the scattering problem. This is experimental evidence that two electrons scatter each other in the head on collision and the scattering cross section is finite. Because electron is Fermion and two particles 
can't stay in the same phase space. On the other hand there is no report on the finite cross section of two photons head on collision, because it is Boson and can stay in the same phase space according to the Pauli's exclusion principle. Photon is truly a wave as we know as a radio wave. We should know that the meaning of the wave nature is different for Fermion and Boson.

We know that we can't form laser by electron, because it is Fermion, but we know that the ensemble of Fermion can form a Boson and leads to a laser. We shouldn't, however, call this ensemble the wave. We are not sure whether every such ensemble stays in the exact same phase space or not. We understand that it is a train of ensembles aligned in an equal spacing. Hereby we understood the real meaning of Pauli exclusion principle that Fermion is always an individual particle and Boson is always a wave. We know that the particle nature of the photon can be interpreted as a wave packet in the wave dynamics. Thus it is not necessary to think that Boson is a particle. We can understand the particle and wave in a classical concept, since it is always a particle or wave.

\section{Uncertainty}

\subsection{Heisenberg's Thought Experiment Demonstrates that Electron Is a Particle}

Uncertainty principle is based on the wave nature of particle, and if uncertainty is broken, this must be another evidence of author's claim that Fermion is always a particle.

The Heisenberg's thought experiment that is a measurement of electron momentum and position by optical microscope is actually the case of the famous Compton scattering. An electron and a photon can be treated as a particle, and we calculate the momentum and energy of both scattered photon and electron by kinematics. Heisenberg treats the photon as a classical wave, and introduces the diffraction limit. When the microscope is used, the optical aperture has to be certainly wide to avoid the occurrence of diffraction, which introduces uncertainty of momentum. If we, however, use an ideally small scintillation detector instead of the microscope, no interfereence should appear. If we use a cloud chamber under magnetic field, we can measure exactly the electron position and momentum simultaneously. In the high energy physics we always measure accurately the momentum and energy of elementally particles. Do you think this is because the energy is high, and the wavelength is short? No we can always measure the momentum and position accurately as Hasegawa group measured in the low energy neutron case. In the Heisenberg thought experiment the uncertainty never appears. This indicates that electron is a particle. If so question arises. When and how the uncertainty principle appears? This must be an important question to understand the foundation of quantum mechanics.

\subsection{Uncertainty Appears when Elementary Particles Are Captured}

Author thinks that uncertainty appears only when Fermions are captured in a potential [12]. The captured electrons shows particular distributions in a closed system like atom, which is solved by the Schrödinger's equation. Neutron or proton behaviors in a nucleus are also the problem of the closed system. Electrons or neutrons stay in a particular state and level in the closed system. This is the observed phenomena. These levels have a certain band width, which determines the uncertainty. The lifetime of the level $\Delta \tau$ and the band width $\Delta \varepsilon$ follows the uncertainty principle, $\Delta \tau * \Delta \varepsilon>h / 2 \pi$ as well known. Uncertainty remains in nucleus and atoms. These cases are indeed the intrinsic quantum mechanical uncertainty. Nobody can predict when radio isotope decays, or when photon will be emitted from an atom. This is the real meaning of uncertainty.

Then we answer to the double slit problem [13]. Tonomura believes that electron passed two slits simultaneously, thus it must be a wave. It is clear for the electro-magnetic wave in the double slits problem. We can say that wave passed the both slits simultaneously. If we express this phenomenon by the word photon, we can say that photons are localized at the detector after passed two slits in a particular density distribution. This we call the wave nature. Not only in the double slits but also in a cavity localization of photon appears. Photon shows the uncertainty and diffraction leading to the mode with particular distribution statistically.

How about the case for electron? We think that the double slits problem is also the case of captured particle regardless of electron, neutron or photon. See Figure 3. There, an electron gun, or photon or neutron sources, double slits or mirrors, and an imaging device, compose the closed system or cavity. When one of the slit is closed the interference pattern on the imaging device disappears. We understand that when one of the slit is closed the system turns to the open cavity, and then the interference pattern disappears. As far as electron or neutron stays in the region A or B of cavity with both slit open, we will see the interference pattern, but if one of the slit is closed the interference pattern disappears, even if it is closed when particle is in the region B. Seems timing has no important role. The important thing is that particles are captured or not. This will be the key in this problem. When particles are captured in a cavity as well as in an atom or a nucleus, mode appears and localizes by the interference.

If electron is an individual particle as described in the 


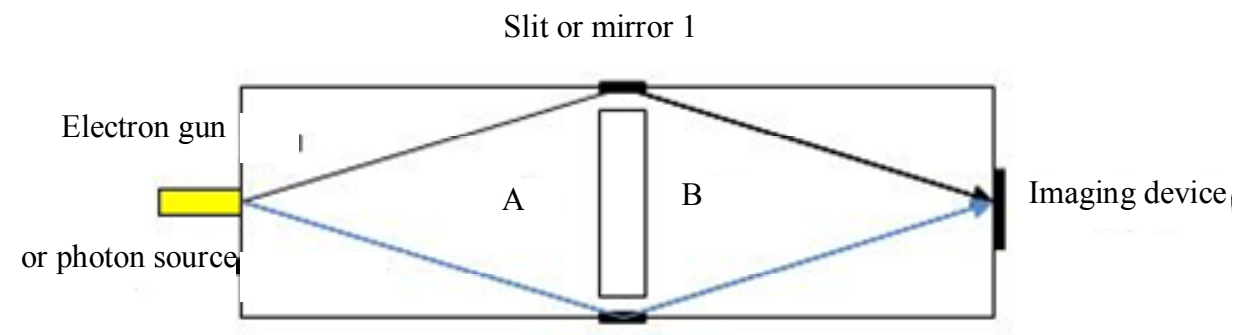

Slit or mirror 2

Figure 3. Double slit problem is equivalent to the closed system or cavity. Electron gun, slit or mirror 1 and 2, and imaging device form a cavity. When particles are captured in the closed system, interference appears. No matter when one of the slit is closed, it makes the open cavity and the interference disappear. This is same for macroscopic as well as microscopic problems.

Section 3, people think that electron cannot split into two, but author say that electrons are the individual body which interfere with other electrons. Many other electrons are there in the slits, mirrors, imaging device and electron gun composing the double slit problem. Let's think that one electron is emitted from the gun and travels in the "field" created by many other electrons in this cavity. This "field" is changed by closing or opening the slits. This field might not be an electro-magnetic field, but might be a "Schrödinger's field" or "quantum field" which has a structure of Hilbert space. The traveling electron feels the change in the "Schrödinger field" and decides where to go on the imaging device. This is the possible interpretation when electron is the individual body and not wave. Wave nature is represented by the "Schrödinger's field". Schrödinger's equation descries the Schrödinger's field, but not the electron wave. Indeed the wave function actually defines the state or configuretion of electrons. We should call the state function instead of the wave function. We discuss more about the meaning of the Schrödinger's equation in Section 5.

Hereby we understand the existence of one electron is defined by all other electrons surrounding and forming the circumference of this electron. This is the real meaning of existence. This electron behaves differently in the different circumference changed by opening or closing the slits. The path of this electron is influenced by all other electrons, and finally decides the location in the imaging device to form the well known interference pattern.

Let's say Fermion is an individual particle which interfere each other. "Interfere" means the quantum mechanical interference described in the wave function, and is different from interaction. We know the word interference, which is used for the relation between humans. Electron has a nature which interfere each other like humans. How interfere is different for electron and human, but the concept of interference is same. Human changes its position and momentum due to the appearance of other individuals. It is the same for electron. Nobody can expect the others position to be there. Nobody knows what the next motion of human will be exactly. It is the same for electrons. Electrons as well as humans behave as they have a sort of "will" [6,10-12]. Electron has a freedom to decide his position under constrains such as the energy conservation low, the momentum conservation low, and the principle of least action. This is the same for human body. Human has to follow the same regulation which electron follows. In addition human has to follow regulations of societies.

The above described is a common nature in both microscopic as well as macroscopic world. Humans and electrons follow the similar regulation, and both are the individual which interfere each other. It is for sure that we don't call that human is a wave, and electron is as well. Why every individual interferes? This is the important question to be answered, instead of the discussion which slit has electron passed.

Interference appears when electron or neutron interferes with other electrons or neutrons composing the cavity. No matter how the cavity is small like atom or large like the double slit problem. Interference is a nonlocal phenomenon. We know the wave function how it spread. If only one electron exist in the universe, this wave function spread all over the universe.

In the word interference we expect that the meaning "recognize", "communicate", and "influence" are included, and is a nonlocal phenomena. We may apply such meanings to the word interference in quantum mechanics [10-12]. As a natural consequence electron must recognize the number of slits before traveling into the slit, even recognize the closed slit afterword in the region B, because the "state function" or "Schrodinger field" spread all over in the cavity. Then in the cavity particular density distribution appears, and makes the interference pattern on the imaging device.

\subsection{Another Thought Experiment}

Let's think another though experiment which represent another digit evidence of "Fermion individual particle 
theory".

In the double slit problem of Figure 3, if two electrons are injected to this cavity simultaneously what will happen. Author thinks that the interference pattern will disappear. If electrons are truly wave, each electron path through independently the both slits simultaneously and creates the interference pattern as indicated in the Figure 2.

When we however follow the Fermion individual particle theory, the case more than two particles are injected simultaneously, one particle to the slit 1 , and another particle to the slit 2, we shouldn't see the interference patter. Each particle recognizes each other, the "quantum field" generated by many other particles composing the cavity will be distorted by another particle. This "quantum field" is shared by two or many particles like in an atom, and the space is shared. In each hemisphere only one slit is there and the interference pattern will be different from the case that the double slits are recognized. If photons are used the result is obvious that the interference pattern is same for both cases, two photon and one photon cases since each photon occupy the whole cavity quantum filed as they are a Boson and truely a wave. We can prove in this way that Fermions are the individual body and exclusive to others, which is the most important features of the particle nature. This experiment proves that Fermions propagate as a particle and detected as a particle. We will see that the wave like nature is different for Fermions and Bosons.

\section{What Is the Meaning of the Schrödinger's Equation?}

What is the meaning of the Schrödinger's equation if electrons are particles and individual bodies, which interferes each other? Author thinks that the wave function represents the Schrödinger or quantum field where the interference among individuals appears [12]. Wave function represents the state of particles. We can call it the state function. Schrödinger's equation can be named as interference equation.

We can interpret the meaning of $\Psi \Psi^{*}$. The complex conjugate of $\Psi, \Psi^{*}$ is the field generated by other individuals which are recognized as others under consideration. The $\Psi^{*}$ is a time reversal but not a signal from future. It is a sum of the reflections from all other particles. The $\Psi \Psi^{*}$ express the density distribution in the steady state after the communication is finished between individuals engaged in this problem. By the communication individuals changes their position and shows particular distribution, which is observed for instance as shown in Figures 1 and 2. We understand here that the existence of one electron is defined by other electrons surrounding. This is the meaning of existence in the microscopic world. This definition of existence is also applicable to macroscopic world and human body. My existence is determined by you and other human. This philosophy is also applicable to the microscopic world.

Hereby the meaning of the electron existence is defined quantum mechanically. Consequently our human existence is also defined by the quantum mechanics. The existence must be an individual body which interfere each other. This definition of existence is different from either that of the philosophy of materialism or that of idealism. Materialism and idealism are combined in the definition of quantum mechanical existence. We may call this new philosophy the quantum mechanical materialism.

We can now understand the QM by natural human sense or common philosophy. By this philosophy we learn more about the Schrödinger's equation. It is applicable to economy and sociology too. We treat human as individual which interfere each other as same as electrons. DNA, protein, chromosome, bacteria, cell, fungi, insect, plant, animal for instance are also same individuals. We may treat planet, galaxy as the body interfere each other by gravity, although the interference occurs in extremely slow manner as we human can't recognize. This is our world. Interference is the key word. We should introduce "interference" in the foundation of physics, and then we can expand the application field of Schrödinger's equation to dynamics of protein, sociology and economy. Every individual interfere each other and then we can describe the relationship by the framework of Schrödinger's equation. We can introduce the "potential" in the field of economy and sociology which is followed by statistics and probability of money flow or human flow. We know that the potential is made of massive humans as well as massive electrons.

Now the question is the relation between "will" and "interference". The free will theory is introduced in the reference 6 . In this article Conway and Kochen didn't discuss the meaning of will, didn't assume that only an individual particle has a free will, and discussed that all elementary particle has a free will. But to introduce the word will, we need the concept of individual which associates the will.

We could approach to the meaning of "will" in this paper. We might say that the individuals which interferes each other, recognize others, and influence others have a will. (Yamada proposed the word will in Japanese, 意志 (pronounce Ishi) in 1996 to explain the meaning of quantum mechanics [10], and published in English in 2004 [11].) We can use the word "will" in a limited definition. The will is the substance, which keep the individual body integrated or united, which recognizes other individuals, and which influence others and changes others state. Here each individual has each will, cooperate, 
and make the world balanced. This is the same for every living specimens as well as electrons. Let's introduce the concepts individual, interference, quantum field and will in the foundation of physics. In Japanese there is a word $\mathrm{Ba}$, which is translated to field but the meaning is more like relationship. The $\mathrm{Ba}$ will be a suitable word for the quantum field or Schrödinger's field.

We could have a new and natural insight into the Schrödinger's equation. The Schrödinger's equation defines the quantum field or say Schrodinger's field in which particles interfere and communicate each other. We don't need to assume that Fermions itself are wave. Schrödinger's equation solves the relationship between electrons. Author calls Schrödinger's equation the dialogue or interference equations. If so we should be able to apply the Schrödinger's equation to not only for elementary particles but also to human society and any living individuals interfering each other.

\section{Concluding Remarks}

Real meaning of Schrödinger's equation is revealed by the fact that electron is always a particle.

In this paper author discussed that Fermion is always a particle and Boson is always a wave by reconsidering past experimental evidences and Pauli Exclusion Principle. We discussed that more than two individual Fermions as well as humans can't stay in the exact same phase space. Herein the meaning of the particle is an individual body. Individuals can't merge each other but photons can merge each other since it is a wave or field. Experimental evidence in which Fermions scatter each other but photons never scatter each other is very important evidence that Fermions are individuals such as human bodies.

In the double slits problem, author could explain how individual particle make interference pattern on the imaging device without assuming that this particle passed two slit simultaneously. Interference appears when this particle interferes with all other particles composing the gun, slits, and detector. Interference appears when the particles are captured in a cavity or potential and interferes with particle composing the cavity or potential. Particle travels the "quantum field" composed by many particles around one. "Quantum field" is different for the two slits open and one slit open cases then this particle recognize the difference of the two quantum fields and behave differently.

Interference is the key word. Interference is the word to describe our living nature as well as the quantum mechanical phenomena. Interference is a word which defines the relation between living individuals. We may include the Fermion in the category of such individuals. Thus without other particles appearance interference never appears. The particle traveling in a free space never shows uncertainty as concluded by Hasegawa and Ozawa.

We can say that the uncertainty appears by the "will" of electron. As we understood QM in the natural commonsense we are able to use the QM more freely. QM defines how interference appears among individuals. Then we learn how to apply and what sort of problems are in a category to be solved by QM.

The meaning of Schrödinger's equation is now clarified. The wave function deals the state of particles, but not the Fermion wave. It can be called as the state function that represents the structure of "quantum field" or Schrödinger field. The $\Psi \Psi^{*}$ defines the existence. The $\Psi^{*}$ is the message from all other particles surrounding the particle in the problem. One's existence is defined by all other individuals. Consequently we learn that the meaning of existence is same for electrons as well as human. This is one of the important conclusion drown by this paper that QM deal with the real existence or real material which you can touch. QM is the tool to study not only the elementary particles but also human body or human society and living specimens.

Author believe that the problems raised by Bohr, Schrödinger and Einstein are solved by the Fermion individual particle theory. Fermions are always particle and no need to assume that the wave turned to the particle by observation. No need to think that the shrink happened in the human brain. Observation is an action taken by human body, thus human interfere the observation. Quantum mechanics define the existence. No need to worry the existence of moon without seeing. Moon existence is defined by many other individuals including electrons as well as human.

\section{REFERENCES}

[1] A. Einstein, B. Podolsk and N. Rosen, "Can QuantumMechanical Description of Physical Reality Be Considered Complete?" Physical Review, Vol. 47, No. 10, 1935, pp. 777-780. doi:10.1103/PhysRev.47.777

[2] E. Schrödinger, "Die Gegenwärtige Situation in der Quantenmechanik," Naturwissenschaften, Vol. 23, 1935, pp. 807-812.

[3] A. Aspect, J. Dalibard \& G. Roger, "Experimental Test of Bell's Inequalities Using Time-Varying Analyzers," Physical Review Letters, Vol. 49, No. 25, 1982, pp. 18041807. doi:10.1103/PhysRevLett.49.1804

[4] J. Erhart, S. Sponar, G. Sulyok, G. Badurek, M. Ozawa and Y. Hasegawa, "Experimental Demonstration of a Universally Valid Error-Disturbance Uncertainty Relation in Spin Measurements," Nature Physics, Vol. 8, 2012, pp. 185-189.

[5] Heisenberg, "Uncertainty Principle." http://en.wikipedia.org/wiki/Uncertainty_principle

[6] J. Conway and S.Kochen, "The Free Will Theorem," 
Foundations of Physics, Vol. 36, No. 10, 2006, pp. 14411473. doi:10.1007/s10701-006-9068-6

[7] M. F. Crommie, C. P. Lutz and D. M. Eigler, "Confinement of Electrons to Quantum Corrals on a Metal Surface," Science, Vol. 262, No. 5131, 1993, pp. 218-220.

[8] Tonomura, "Electron Waves Unveil the Microcosmos," Friday Evening Discourse at Royal Institution, 1994.

[9] Pauli Principle. http://en.wikipedia.org/wiki/Pauli_exclusion_principle

[10] H. Yamada, "Quantum Theory Named Dialog Principle,"
Soryusiron, Vol. 94, No. 2, 1996, pp. 53-68.

[11] H. Yamada, "Quantum Mechanical Approach to the Meaning of Existence, Will and Life," AIP Conference Proceedings, Vol. 716, 2004, pp. 109-115.

[12] H. Yamada, "Ryousi Rikigakuga Akirakanisuru Sonzai, Ishi, Seimei Noimi," Koushiken Publish, Kusatsu, 2011, pp. 1,172 .

[13] C. Jönsson, "Electron Diffraction at Multiple Slits," American Journal of Physics, Vol. 4, No. 1, 1974, pp. 411. doi:10.1119/1.1987592 